\title{
Impact of Pharmaceutical Care Services in Health Related Outcomes among Children with Thalassemia
}

\author{
Almana Rodrigues ${ }^{1}$, Jitha $Y^{1}$, Merlin Susan Paul ${ }^{1}$, Sajja Prabhatha \\ Lakshmi $^{1}$, Juny Sebastian ${ }^{1 *}$ and Mandyam Dhati Ravi ${ }^{2}$ \\ ${ }^{1}$ Department of Pharmacy Practice, JSS College of Pharmacy, JSS Academy of \\ Higher education and Research, Mysuru, Karnataka, India \\ ${ }^{2}$ Department of Paediatrics, JSS Medical College and Hospital, JSS Academy of \\ Higher education and Research, Mysuru, Karnataka, India \\ *Corresponding Author: Juny Sebastian, Lecturer, Department of Pharmacy \\ Practice, JSS College of Pharmacy, JSS Academy of Higher education and Research, \\ Mysuru, Karnataka, India.
}

Received: May 10, 2021

Published: May 17, 2021

(C) All rights are reserved by Juny Sebastian., et al.

\section{Abstract}

Background: Thalassemia is a rare disease that pose significant morbidity among the affected individuals due to various reasons such as drug related problems (DRPs), poor knowledge, attitude and practice (KAP) of thalassemia disease and its treatments. These unresolved problems lead to poor medication adherence and there by compromise in quality of life among the thalassemia patients specially children. The current study was conducted to assess the impact of pharmaceutical care services in health related outcomes among Thalassemia patients.

Methods: This was a randomized controlled study conducted at Hematology Clinic, JSS Hospital Mysuru. Eligible subjects were enrolled after taking informed consent randomized to test and control groups. Study participants medical records were assessed for actual or potential DRPs, KAP on thalassemia, HRQoL and medication adherence during the first visit (V1). Standardized education by clinical pharmacist was provided to the test group participants during all the study visits (V2 and V3) while control group participants received a validated information leaflet during first visit (V1) and no further interventions in subsequent visits (V2 and V2). At the end of the study, study participants of both the groups were reassessed for DRPs, KAP, HRQoL and medication adherence. Results: A total of 48 subjects were consented for the study. The study could significantly decrease the DRPs of the study population in the test group. Also, KAP ( $p$ 0.001), HRQoL ( $p$ 0.001) and medication adherence (p 0.001) of the test group was significantly improved when compared to the control group study population.

Conclusion: Practicing clinical pharmacists can significantly influence the health related outcomes children with thalassemia by providing different pharmaceutical cares services and individual education to the children and their LAR.

Keywords: HRQoL; KAP; Medication Adherence; PedsQL; Pharmacist's Education; Thalassemia Patients

\section{Introduction}

Thalassemia is a group of hereditary blood disorders that are characterized by reduced or absent globin chain synthesis, imbalances of globin chains cause haemolysis and impair erythropoi- esis, resulting in reduced haemoglobin, decreased RBC production leading to anaemia. It affects approximately 4.4 of every 10,000 live births and around 3,50,000 births/year with severe hemoglobinopathies are reported worldwide [2]. Thalassemia is caused 
mainly due to the mutations in the genes that form haemoglobin. It disrupts the normal production of healthy red blood cells causing anemia leading to oxygen deprivation to tissues [1]. There are two types of Thalassemia: Alpha thalassemia and Beta thalassemia [3]. In alpha thalassemia, the alpha genes are affected, which most commonly are presented through gene deletions. Abnormal production of alpha-globin chains results in excess of gamma-globin chains in fetuses and newborns and of beta-globin chains in children and adults [4]. $\beta$ thalassemia occurs when there is a quantitative reduction of $\beta$ globin chains that are usually structurally normal [2]. It is caused by mutations that affect the $\beta$ globin locus and are extremely heterogeneous.

The symptoms vary depending on the type and severity of condition. There are bone deformities, especially in the face, dark urine, delayed growth and development, excessive tiredness and fatigue, yellow or pale skin [6]. The treatments are regular blood transfusions and stem cell transplant. The frequency of transfusion is every two to four weeks. The amount of blood to be transfused depends on several factors including weight of the patient, target increase in Hb level and hematocrit of blood unit. Regular blood transfusions can cause an overload of iron in blood, which can damage heart, liver and other organs; which can be prevented and treated with iron chelating therapy such as deferoxamine, deferasirox, deferiprone [7]. The intensive demands and uncomfortable side effects of therapy can have a negative impact on daily activities and well-being, which may affect adherence. Due to various problems such as frequent hospital visits for transfusion, absence to the schools and the transfusion related complications also negatively affect the QoL of thalassemia specially in pediatric patients. The possibility of drug related problems such as sub therapeutic dose, adverse drug reactions, failure to receive drugs and associated medication errors are also some of the contributing factors which affects the medication adherence among thalassemia patients and there by the depressed QoL [4].

There are different studies showed that the patients with thalassemia are possessing low quality of life due to various problems and it can be overcome training by the health care professionals. Also, studies are confirming that the improvement in their knowledge and attitude can influence the HRQoL of the thalassemia patients
[2]. Hence the study was aimed to assess impact of pharmaceutical care services and the individualized education in KAP, HRQoL and medication adherence among children with Thalassemia.

\section{Materials and Methods}

- Study design: Randomised controlled study.

- $\quad$ Study site: Hematology Clinic at Pediatric Department of JSS Hospital, Mysuru.

- $\quad$ Study period: Six Months (October 2018 to March 2019).

- Study population: Children diagnosed with Thalassemia.

- Study criteria: Patients with an established diagnosis of thalassemia and visiting the study site for regular red blood cells transfusion was included in the study. Patients with any associated chronic disease not related to thalassemia or its complication was excluded from the study.

- $\quad$ Ethical clearance: Study was approved by the institutional ethical committee of JSS College of Pharmacy, Mysuru on $30^{\text {th }}$ October 2018.

Study tools

- Questionnaire to assess the KAP of thalassemia patients.

- $\quad$ Patient information leaflet (PIL).

- Questionnaire to assess the health related quality of thalassemia parents: PedsQL 4.0 Generic Core Scale Questionnaire.

- Morisky-Green-Levine Scale.

Development and validation of questionnaire to assess KAP of thalassemia patients

KAP questionnaire was developed after an extensive literature search using key words such as thalassemia, beta thalassemia, KAP of thalassemia patients, validation of questionnaire, content validity index using search engines like Google scholar, Science Direct, PubMed/Medline and Clinical Key. The questionnaire was developed in English and face validity was performed with the help of experts practicing in Pediatrics. The research team finalized questionnaire after a detailed discussion taking the feedback received from experts into consideration. The questionnaire consisted of 19 questions, including 11 questions that sought to gain insight into the parents knowledge, 5 questions related to attitude and 3 questions related to practice towards thalassemia and its management. 
Experts in the related field validated the questionnaire using the Content Validity Index developed by Waltz and Bausell. The experts rated each question and answer for clarity, relevance, ambiguity and simplicity. The scale content validity index (S-CVI) was calculated using the questionnaire rating by the experts the collected data was entered into Excel spreadsheet for easy calculation and retrieval followed by the analysis using statistical package for social studies (SPSS) software version 22 (licensed to JSS University). The reliability/internal consistency of each question in the questionnaire was estimated using Cronbach's Alpha (102). The final questionnaire was translated to the local language and back translated to English by the bilingual expert for the use in the study.

\section{Patient information leaflet}

The leaflet was prepared in English based on an extensive literature search using key words such as Thalassemia symptoms, Thalassemia management, Thalassemia complications using search engines like Google scholar, Science Direct, PubMed/Medline and Clinical Key. The developed PIL had details of thalassemia disease, medications, importance of blood transfusions and complications of disease. The leaflet was then reviewed by the experts in the same field. PIL was translated to local language and back translated for the use in the study by a bilingual expert. PIL was validated for its readability with the help of Flesch Readability Ease(FRE) formula, Quality of information using Ensuring Quality Information Patients (EPIQ) and layout and design with the help of Baker Able Leaflet Design (BALD) criteria.

\section{PedsQL 4.0 generic core scale questionnaire}

The Pediatric Quality of Life Inventory (PedsQL ${ }^{\mathrm{TM}}$ ) 4.0 version measurement model is a modular approach developed by James.W.Varni, Mapi Research Trust to measure Health-Related Quality of Life (HRQoL) in healthy children with acute and chronic health conditions [9]. The researcher group received the permission to use the PedsQL $L^{\mathrm{TM}}$ in the to assess the HRQoL for the study population. The questionnaire consisted of different sections to assess the HRQoL with reference to domains such as Physical, Emotional, Social and School. Physical domain consisted of 8 questions, emotional domain consisted of 5 questions, social domain consisted of 5 questions and school domain consists of 5 questions. The number of questions answered for never a problem has a score of 0 , almost never a problem has a score of 1 , sometimes a problem has a score of 2 , often a problem has a score of 3 and almost always a problem has a score of 4 .

\section{Morisky medication adherence scale (MMAS-4)}

Morisky medication adherence scale (MMAS-4) is a 4-item scale used to assess the medication adherence. The four items consisted of "yes" or "no" responses to each question. The number of questions answered with "yes" determined the level of medication adherence. If all questions are answered with "no", the patient is assessed to have high medication adherence. If 1 of the four questions was answered "yes", the medication adherence was considered to be moderate. If "yes" is answered for two or more questions, then the patient is considered to have low medication adherence.

\section{Study procedure}

Eligible subjects were enrolled after taking the informed consent (based on their language preference) from their parents/legally acceptable representative (LAR). Informed consent form was prepared in English as per the Indian Council of Medical Research (ICMR) guidelines [10] and was translated to local language. Required data such as subject's demographic details, contact details, laboratory reports, past medical history and thalassemia management was collected and documented in a suitably designed data collection form. Enrolled subjects medical records were reviewed thoroughly and looked for any actual or potential DRPs. Appropriate interventions were made to the treating paediatrician on identification of the DRPs and educated the subjects on the same. Baseline KAP, HRQoL and the medication adherence were assessed for the entire study population using the study tools. The subjects in the test group and their parents/LAR received standard education on thalassemia and its management by the research team during the first visit (Visit1). A follow-up education was provided to the test group subjects and their parents again on the second and third visits which were scheduled with a gap of 3s. KAP, HRQoL and medication adherence was reassessed for the entire study population during their fourth visit. After the assessment, the control group received standard education on thalassemia and its management as received by test group.

\section{Data analysis}

The collected data was entered into Excel spreadsheet for easy calculation and retrieval followed by the assessment with the help of SPSS software version 22. Quantitative data was expressed in mean, standard deviation and percentages. The bar diagrams were used appropriately. Median split method was used to categories the Knowledge, Attitude and Practice (KAP) of study population into adequate and inadequate. Comparison of mean scores between the control group and intervention group were performed using independent sample $t$ test. Comparison of pre and post knowledge, attitude, practice and total KAP, HRQOL and medication adherence were done using paired sample t-test. A 'P' value of $<0.005$ was considered as statistically significant with $95 \%$ confidence interval. 


\section{Results}

\section{Study tools}

The average content validity index score of the questionnaire to assess the KAP of thalassemia patients was $81.2 \%$. As the score was more than $80 \%$ none of the questionnaire was deleted from the prepared questionnaire and used as a standard tool to assess the KAP of the study population. Readability score of the developed PIL assessed using FRE was 98.05. The score of 24 was obtained for the layout and design of the PIL using BALD Criteria. The assessed quality of the information present in PIL, EQIP score was $86.1 \%$.

\section{Demographic details of study population}

Out of 60 patients visiting the study site, 48 eligible subjects were enrolled and randomised into test $(n=23)$ and control ( $n$ $=25$ ) group using block randomisation technique. Among the study population, $66.66 \%$ were male gender and $33.34 \%$ were female gender, $22.9 \%$ study population were between age group 0 - 5 years, 39.58\% were between 5-10 years, $22.9 \%$ were from between 10 - 15 years and $14.62 \%$ of population was from between $15-20$ years, $45.84 \%$ were living in rural area and $39.58 \%$ belonged to socioeconomic class of upper middle as per the Kuppuswamy socio-economic classification.

\section{Drug related problems}

A total of 35 and 28 drug related problems were identified in test group and control group respectively during the treatment chart review of enrolled subjects in the beginning of the study. Test group DRPs included 12 Adverse drug reactions, 11 drug - drug interactions, 3 sub therapeutic doses, 4 drug use without indication and 5 failure to receive drugs. Appropriate interventions were made to the treating pediatrician and the DRPs were rectified. Also, educated the test group subjects on about the precautions to be taken to avoid ADRs such as gastrointestinal related reactions while receiving deferasirox, how to space the drugs to prevent drug interactions and measures to improve the failure to receive drugs. At the end of the study, DRPs were decreased to 11 among the test group subjects.

Out of 28 DRPs identified among the control group subjects, 11 were ADRs, 10 were drug interactions and 7 were failure to receive drugs. DRPs were communicated to the treating pediatrician and the subjects and their LAR. But the number ADRs didn't decrease by the end of the study, but increased to 30 (added 2 more failure to receive drugs).

Knowledge about the disease in thalassemia

Test group participants have shown improvement in the knowledge on thalassemia and its management at the end of the study. There was $59.13 \%$ of improvement in the knowledge of curability of thalassemia in the test group at the end of the study whereas control group showed an improvement of only $8.0 \%$. Similarly all of the test group participants were knowing the type of thalassemia at the end of the study where as $12 \%$ in the control were not knowing the same even at the end of the assessment. Knowledge on inheritance of thalassemia, its risk factors and about the need of blood transfusion was improved by $48.22 \%, 65.7 \%$ and $21.74 \%$ respectively in the test group by the end of the study. Both the test group and the control group participants knew the need for using iron chelating therapy among thalassemia patients at the end of the study. The complete information on various knowledge related questions and its answers are presented in table 1.

\begin{tabular}{|c|c|c|c|c|c|c|c|}
\hline \multirow{2}{*}{ Knowledge Questions } & \multirow{2}{*}{ Group } & \multicolumn{3}{|c|}{ Pre Intervention (\%) } & \multicolumn{3}{|c|}{ Post Intervention (\%) } \\
\hline & & Yes & No & Don't know & Yes & No & Don't know \\
\hline \multirow[t]{2}{*}{ Is thalassemia a curable disease? } & Test & 39.14 & 26.08 & 34.78 & 14.79 & 85.21 & 00 \\
\hline & Control & 16 & 40 & 44 & 36 & 48 & 16 \\
\hline \multirow[t]{2}{*}{ Do you know the different type of thalassemia? } & Test & 39.13 & 13.04 & 47.83 & 100 & 00 & 00 \\
\hline & Control & 32 & 24 & 44 & 88 & 12 & 00 \\
\hline \multirow[t]{2}{*}{ Is thalassemia a inherited disease? } & Test & 23.91 & 30.45 & 45.64 & 72.13 & 27.87 & 00 \\
\hline & Control & 8 & 24 & 68 & 20 & 60 & 20 \\
\hline \multirow{2}{*}{ What are the risk factors for development of thalassemia? } & Test & 26.3 & 45.31 & 28.39 & 92 & 6 & 2 \\
\hline & Control & 32 & 56 & 12 & 40 & 40 & 20 \\
\hline \multirow{2}{*}{$\begin{array}{l}\text { Thalassemia patients should receive blood transfusion } \\
\text { throughout life? }\end{array}$} & Test & 65.21 & 15.23 & 19.56 & 86.95 & 6.52 & 6.52 \\
\hline & Control & 84 & 16 & 00 & 90 & 10 & 00 \\
\hline \multirow[t]{2}{*}{ Is Iron chelating therapy essential for a thalassemia patients? } & Test & 69.56 & 4.34 & 26.08 & 100 & 0 & 0 \\
\hline & Control & 84 & 8 & 8 & 100 & 0 & 0 \\
\hline
\end{tabular}

Table 1: Question and answers to the knowledge questions on thalassemia. 
There was $17.39 \%$ improvement in the knowledge on blood transfusion and as a management strategy in the test group and $3.02 \%$ improvement in the control group at the end of the study. Though 15.2\% answered that they 'do not know' about the treatment strategy for Thalassemia during baseline assessment and none in the test and control group answered the same at the end of the study. Majority of the study population $(86.95 \%$ in test group and $88.0 \%$ in control group) answered that health care professionals are the trustful source of information about the disease and its management followed by internet and social media $(4.6 \%$ in test group and $8.4 \%$ in control group). But at the end of the study more than $90 \%$ in both groups answered that HCPs are the trustful sources regarding any information on thalassemia. $65.21 \%$ in the test group and $60.0 \%$ in the control group gave correct answer on the question on the complication of frequent blood transfusion in the beginning of the study and the all of them answered correctly at the end of the study (Figure 1).
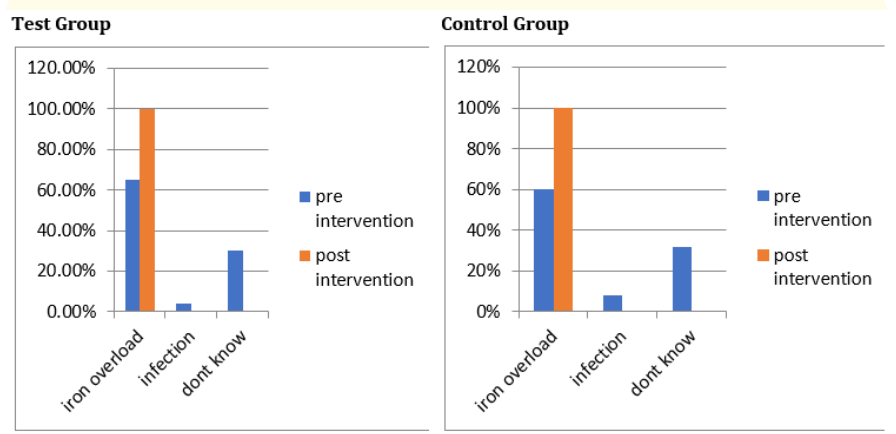

The entire study population (100\%) showed positive attitude towards blood screening for identification of thalassemia, Donation of blood for thalassemia patients and taking part in activities to create awareness about thalassemia among general public in the test group at the end of the study. Practice on taking medicines at home as per the instructions of the pediatrician has improved (100\%) in both test and control group from $91.30 \%$ and $93.22 \%$ respectively. Also, there was an improvement of $30.44 \%$ in the Practice of getting other family members tested for thalassemia in the test group. Complete information on answers of attitude and practice questions of the study population is illustrated in table 2 .

At the end of these study, more than $60 \%$ study population in both test and control group was receiving the blood transfusion without any delat which was $43.47 \%$ and $20 \%$ respectively at the beginning of the study. The details on the practice on blood transfusion is presented in figure 2 .

At the beginning of the study, adequate KAP on thalassemia was observed in $78.26 \%$ population in test group which as increased to $94.30 \%$ after the educational intervention, where as adequate KAP was observed in $77.32 \%$ of population in control group in the beginning of the study which was increased to $80.2 \%$ by the end of the study. There was a statistically significant improvement in the KAP of test group at the end of the study with a P value of 0.001 where as insignificant statics was shown in the KAP of control group. The statistical analysis of the KAP of thalassemia is presented in table 3.

Figure 1: Answers on complication of frequent blood transfusion.

\begin{tabular}{|c|c|c|c|c|c|}
\hline \multirow{2}{*}{ Questions } & \multirow{2}{*}{ Group } & \multicolumn{2}{|c|}{ Pre intervention (\%) } & \multicolumn{2}{|c|}{ Post intervention (\%) } \\
\hline & & Yes & No & Yes & No \\
\hline \multicolumn{6}{|c|}{ Questions on Attitude } \\
\hline \multirow{2}{*}{$\begin{array}{l}\text { Do you think that the Thalassemia } \\
\text { screening blood test is necessary? }\end{array}$} & Test & 95.65 & 4.35 & 100 & 0 \\
\hline & Control & 92 & 8 & 96 & 4 \\
\hline \multirow{2}{*}{$\begin{array}{l}\text { Are you ready to donate blood for } \\
\text { Thalassemia patients? }\end{array}$} & Test & 73.91 & 26.09 & 100 & \\
\hline & Control & 80 & 20 & 92 & 8 \\
\hline \multirow{2}{*}{$\begin{array}{c}\text { Are you ready to take part in any activities } \\
\text { to create awareness about thalassemia } \\
\text { among general public? }\end{array}$} & Test & 86.95 & 13.05 & 100 & 0 \\
\hline & Control & 92 & 8 & 96 & 4 \\
\hline \multicolumn{6}{|c|}{ Questions on Practice } \\
\hline \multirow{2}{*}{$\begin{array}{l}\text { Do you take the medicines at home as per } \\
\text { the instructions of your Paediatrician? }\end{array}$} & Test & 91.30 & 8.70 & 100 & 0 \\
\hline & Control & 93.22 & 0 & 100 & 0 \\
\hline \multirow{2}{*}{$\begin{array}{l}\text { Did you get the others in your family test } \\
\text { for Thalassemia? }\end{array}$} & Test & 69.56 & 30.44 & 100 & 0 \\
\hline & Control & 76 & 24 & 92 & 8 \\
\hline
\end{tabular}

Table 2: Answers on attitude and practice questions on thalassemia. 

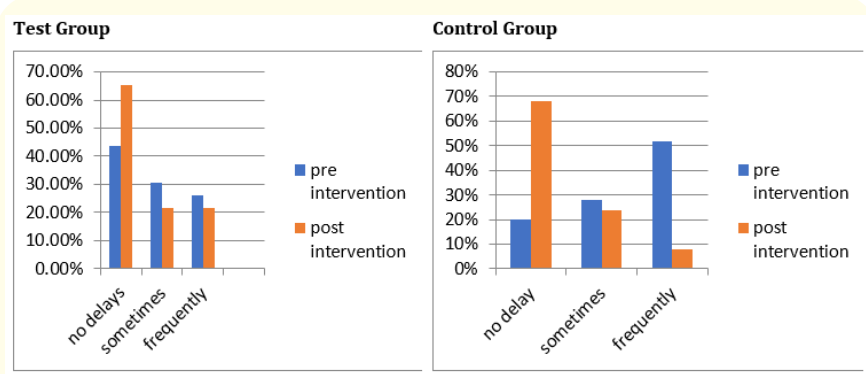

Figure 2: Practice on blood transfusion.
Health related quality of life

Using the PedsQL 4.0 questionnaire, HRQoL was assessed under 4 domains, which were Physical, emotional social and School. There was statistically significant improvement in HRQoL of all domains [physical ( $p$ value -0.001), social ( $p$ value -0.001) and School ( $p$ value -0.001)] except emotional ( $p$ value -0.034 ) in the test group where as non-significant HRQoL scores were observed in the control group at the end of the study. HRQoL scores of test and control groups are presented in table 4 .

\begin{tabular}{|c|c|c|c|c|c|}
\hline \multirow{2}{*}{\multicolumn{2}{|c|}{ Variables }} & \multicolumn{2}{|c|}{ Test Group } & \multicolumn{2}{|c|}{ Control Group } \\
\hline & & \multirow{2}{*}{$\begin{array}{c}\text { Mean } \pm \text { SD } \\
11.20 \pm 4.139\end{array}$} & \multirow{3}{*}{$\begin{array}{c}\text { P value } \\
.001\end{array}$} & \multirow{2}{*}{$\begin{array}{c}\text { Mean } \pm \text { SD } \\
09.46 \pm 4.698\end{array}$} & \multirow{3}{*}{$\frac{\text { P value }}{.086}$} \\
\hline Knowledge score & Pre education & & & & \\
\hline & Post education & $16.98 \pm 3.632$ & & $09.86 \pm 3.935$ & \\
\hline \multirow[t]{2}{*}{ Practice score } & Pre education & $02.54 \pm 0.904$ & \multirow[t]{2}{*}{.024} & $02.24 \pm 1.111$ & \multirow[t]{2}{*}{.272} \\
\hline & Post education & $2.77 \pm 0.565$ & & $02.29 \pm 0.967$ & \\
\hline \multirow[t]{2}{*}{ Attitude score } & Pre education & $03.42 \pm 1.027$ & \multirow[t]{2}{*}{.001} & $03.16 \pm 1.178$ & \multirow[t]{2}{*}{.874} \\
\hline & Post education & $03.82 \pm 0.557$ & & $03.15 \pm 1.009$ & \\
\hline \multirow[t]{2}{*}{ Total score } & Pre education & $17.16 \pm 5.289$ & \multirow[t]{2}{*}{.001} & $14.86 \pm 6.017$ & \multirow[t]{2}{*}{0.327} \\
\hline & Post education & $23.57 \pm 1.584$ & & $15.3 \pm 1.970$ & \\
\hline
\end{tabular}

Table 3: Analysis of KAP of thalassemia patients.

\begin{tabular}{|c|c|c|c|c|c|}
\hline \multirow{2}{*}{ HRQoL Domains } & \multirow{2}{*}{ Study Phase } & \multicolumn{2}{|c|}{ Test Group } & \multicolumn{2}{|c|}{ Control Group } \\
\hline & & Mean \pm SD & $P$ value & Mean \pm SD & $P$ value \\
\hline \multirow[t]{2}{*}{ Physical } & Pre education & $85.31 \pm 4.16$ & \multirow[t]{2}{*}{.001} & $83.80 \pm 4.69$ & \multirow[t]{2}{*}{.036} \\
\hline & Post education & $94.65 \pm 3.62$ & & $86.27 \pm 3.93$ & \\
\hline \multirow[t]{2}{*}{ Emotional } & Pre education & $86.4 \pm 0.82$ & \multirow[t]{2}{*}{.034} & $83.91 \pm 1.32$ & \multirow[t]{2}{*}{.372} \\
\hline & Post education & $91.95 \pm 0.53$ & & $82.00 \pm 0.87$ & \\
\hline \multirow[t]{2}{*}{ Social } & Pre education & $86.95 \pm 1.02$ & \multirow[t]{2}{*}{.001} & $88.20 \pm 1.17$ & \multirow[t]{2}{*}{.874} \\
\hline & Post education & $95.00 \pm 0.55$ & & $90.60 \pm 1.00$ & \\
\hline \multirow[t]{2}{*}{ School } & Pre education & $75.80 \pm 5.28$ & \multirow[t]{2}{*}{.001} & $70.32 \pm 6.01$ & \multirow[t]{2}{*}{0.427} \\
\hline & Post education & $85.21 \pm 4.17$ & & $73.26 \pm 5.91$ & \\
\hline
\end{tabular}

Table 4: HRQoL scores of thalassemia patients.

\section{Medication adherence}

At the end of the study, it was found that the subjects in the test group $(n=23)$ showed high adherence to the medications prescribed by their respective paediatrician, which was increased from the first visit. But the control group study population $(n=3)$ had a medium adherence at the end of the study. The test group study population showed a statistically significant improvement in the medication adherence behaviour (P value - 0.001) specially on use of iron chelating therapy. But statistically significant improvement in the medication adherence (P value - 0.062) was not observed among the control groups. The statistical analysis of scores of medication adherence is presented in table 5 . 


\begin{tabular}{|c|c|c|c|}
\hline Group & Study phase & Mean KAP \pm SD & P value \\
\hline \multirow{2}{*}{ Test } & Pre intervention & $2.78 \pm 0.405$ & \multirow{2}{*}{0.001} \\
\cline { 2 - 4 } & Post intervention & $3.63 \pm 0.355$ & \\
\hline \multirow{2}{*}{ Control } & Pre intervention & $2.62 \pm 0.534$ & \multirow{2}{*}{0.062} \\
\cline { 2 - 3 } & Post intervention & $2.89 \pm 0.453$ & \\
\hline
\end{tabular}

Table 5. Pre and post education scores of medication adherence.

\section{Discussion}

Study enrolled less number of study population (48 Thalassemia Patients), where as previously conducted study assessing the KAP of Thalassemia patients [14] had a large sample size (n $=428$ ) as the later study was conducted by enrolling population attending general out patient department. The knowledge on curability was known by $26.08 \%$ and $40.0 \%$ in test group and control group respectively which as increased to $85.21 \%$ and $48 \%$ by the end of the study. In the study by Dr. Basu., et al. also found a similar result (48.37\%) about the curability of the thalassemia disease as seen during the beginning of the study [11]. Almost similar result as seen in the beginning of our study is observed for the various similar knowledge questions such as preventability of thalassemia (69.19\%), treatment of thalassemia specially blood transfusion (66.59\%) in the study by Basu., et al. [11]. This similarity in the knowledge on thalassemia shows that the study population did not have any extra knowledge on thalassemia and its management and their knowledge level as similar as any general population.

Ishaq F [23] showed that $44.6 \%$ were aware of its types after intervention. The other question was inheritance of thalassemia which was compared with the study conducted by Dr. Basu.M [11] showed that $60.05 \%$ were aware that thalassemia is inherited disorder which gave a better result in this study. Requirement of blood transfusion in thalassemia was compared with the study conducted by Saxena.A [12] showed 90\% study population agreed. A study conducted by Han K.E [10] showed that $55 \%$ of study population were not aware about the risk factors of thalassemia which was $2 \%$ post intervention in this study. The study conducted by Saxena A., et al. [12] showed that $92.5 \%$ of subjects responded positively for chelating therapy, there was also a study conducted by Basu M [11] which showed that $27.73 \%$ of study population were aware of the treatment options, the other question related to the knowledge on sources of information $37.8 \%$ got information about thalassemia by Health care professionals, $31.77 \%$ by internet, $6.77 \%$ by seminars and lectures and $29 \%$ by friends and relatives and a final ques- tion which was considered from the same study was the knowledge on possible complications of frequent blood transfusion in thalassemia which showed $27 \%$ which gave a better results in this study [19].

Considering the questions related to attitude of subjects on necessity of thalassemia screening blood test the results were $100 \%$ post intervention in this study, the second question was on the donation of blood for thalassemia patients which also gave a positive attitude among the parents and study participants, and the final question was to take part in awareness activities about thalassemia in general public in which it increased $96 \%$ of study participants agreeing for the same. This study was compared with the study conducted by Moghaddam E.M [24] showed (78.6\%) positive attitude regarding thalassemia. Practice questions included taking medications as per the instructions of the paediatrician which showed a significant improvement post intervention in both test and control group. The other practice question was to get other family members tested for thalassemia which was $69.56 \%$ and increased to $100 \%$ after the education was provided. All these practice questions were compared with the study conducted by Saxena. A [12] which gave a result of $57.5 \%$.

It is important to assess the health related quality of life in patients and identify the factors, thereby we can improve the quality of life. In our study PedsQL 4.0 questionnaire was used. In that we assess 4 domains which are physical, emotional social and school. The results showed that subjects with thalassemia have significantly higher scores when compared with subjects from control. In a study conducted by Caocci G., et al. [15] showed there is improved QoL in patients who receive conventional iron therapies rather than those who are not on chelation therapy. There were also comparisons with the another study conducted by Wahyuni S.M., et al. [14] showed that there was significant decrease in quality of life parameters compared to this study. Our study indicates a significant value in all the domains in test group compared to control group. The emotional factor was about the psychological behaviour which did not give expected result as compared to the other three domains. The control group did give significant $p$ value as the study participants did not have proper education like that of test group.

Morisky medication adherence scale 4.0 was used in our study. Subjects were counselled about the importance of medications, adverse effects and life style modifications in thalassemia patients, 
then they were assessed using this Morisky medication adherence scale and the results showed that most of the subjects were adherent to the medications. Medication adherence was reviewed in a study conducted by Panda K [13] showed about 7.55 patients are highly adherent, $48.4 \%$ are moderate and $41.9 \%$ were poorly adherent to medications which was considered to be improved significantly in this study.

\section{Conclusion}

A healthy lifestyle is important for everyone. For people living with thalassemia, it is especially important to know that a healthy lifestyle means "managing the disorder", as well as making healthy choices. Around 3,50,000 births/year with severe hemoglobinopathies are reported worldwide [17]. High treatment costs, the use of iron chelators, comorbidities and periodic visits affect the quality of life in patients. Due to the medical advances, regular, safe transfusions and iron chelating therapy, the life expectancy and survival of the patients have increased dramatically compared with previous decades [18].

Parents from different socioeconomic background were targeted in order to get their opinion regarding their health quality and medication adherence. The results of this study revealed a poor health quality of life among the patients while there is significant improvement in knowledge, attitude, practice and medication adherence. Efforts were made to understand the importance of medication adherence in thalassemia patients. Standard educational intervention was provided to the study subjects to improve their overall understanding of their disease.

\section{Acknowledgements}

Authors would like to thank the staff and students of departments of pediatrics and clinical pharmacy, JSS Hospital, Mysuru for their encouragement and support. We also would like to thank the administrators of JSS College of Pharmacy, Mysuru and different schools to grant us permission and technical support to perform the study.

\section{Bibliography}

1. Review of literature. History of thalassemia (2019).

2. Galanello R and Origa R. "Beta- thalassemia". Orphanet Journal of Rare Diseases 11 (2010): 1-15.

3. Yolanda S. "Thalassemia prevalence". News Medical Life Sciences (2019).
4. Babu T., et al. "Prescribing patterns and drug related problems (DRPs) in transfusion -dependent paediatric thalassemia patients: A prospective interventional study from a tertiary care hospital in India". International Journal of Paediatrics and Adolescent Medicine (2020): 1-4.

5. Cheerva CA. "Alpha thalassemia". Medscape.

6. Miller ER. "Beta thalassemia". Kids health from Nemours (2015).

7. Neufeld JE. "Oral chelatorsdeferasirox and deferiprone for transfusional iron overload in thalassemia major". Blood 107.9 (2006): 3436-3441.

8. Ansari SH., et al. "Quality of life in patients with thalassemia major". Iranian Journal of Pediatric Hematology and Oncology 4.2 (2014): 57.

9. Han KE., et al. "Thaleaaemia in the outpatient department of the yangon children's hospital in myanmar. knowledge, attitude and practice in relation to thalassemia". Southeast Asian Journal of Tropical Medicine and Public Health 23.2 (2013): 269-272.

10. National ethical guidelines for biomedical and health research involving human participants. Indian council of medical research. New Delhi: Director-General (2018): 50-55.

11. Basu M. "A study on knowledge, attitude and practice about thalassemia among general population in outpatient department at a tertiary care hospital of Kolkata". Journal of Preventive Medicine and Holistic Health 1.1 (2015): 6-13.

12. Saxena A., et al. "Knowledge, Practice and Experiences of parents with the thalassemic child". International Journal of Contemporary Pediatrics 4.5 (2017): 16302-16333.

13. Panda K., et al. "Adherence to deferasirox among beta thalassemia major children-A cross-sectional study in tertiary care hospital". Deferasirox Adherence Among Thlassemic Children 5.1 (2017): 38-41.

14. Wahyuni SM., et al. "Quality of life assessment of children with thalassemia”. Paediatrica Indonesian 51.3 (2011): 163-169. 
15. Caocci G., et al. "Health related quality of life in middle eastern children with beta thalassemia". BMC Blood Disorder 12.2 (2012): 120-134.

16. Kotzeva A., et al. "Health Related Quality of Life of patients with beta thalassemia". Value in Health 21.3 (2018): 341-381.

17. Ibrahim AY. "Quality of Life Among Thalassemia Children Patients in the Gaza Strip". American Journal of Nursing Science 5.3 (2016): 106-113.

18. Imani E., et al. "Comparison quality of life in patients with thalassemia major based on participating in group activities, Bandar Abbas". Scientific Journal of Iran Blood Transfus Organ 10.2 (2013): 198-206.

19. Shakib AJ., et al. "Health Related Quality of Life in beta thalassemia major children in North Iran". Iranian Journal of Blood and Cancer 8.4 (2019): 108-111.

20. Arian M., et al. "Health Related Quality of Life in beta thalassemia major patients assessed by 36 items short health survey: A meta analysis". Quality of Life Research 28.1 (2019): 321-334.

21. Samsamipour M., et al. "Health Related quality of life in young adult with beta thalassemia major". Scientific Journal of Nursing, Midwifery and Paramedical Faculty 4.4 (2019): 66-75.

22. Smeltzer SC., et al. "Bruner and Suddharth's textbook of Medical-Surgical nursing”. 12.1 (2008).

23. Ishaq F., et al. "Awareness among parents of Beta Thalassemia major patients regarding prenatal diagnosis and pre marital screening". Journal of College Physicians and Surgeons Pakistan 22.4 (2012): 218-221.

24. Moghaddam EM., et al. "High school knowledge and attitude towards thalassemia in Southern Iran". International Journal of Hematology-oncology and Stem Cell Research 8.1 (2014): 2430 .

\section{Volume 5 Issue 6 June 2021}

(C) All rights are reserved by Juny Sebastian., et al. 